\title{
Mono-functionalization on a Foot of Carceplex: An Intermediate for Carceplex Engineering
}

\author{
Chaesang Ihm, Naksin Jung, and Kyungsoo Paek \\ Department of Chemistry, Soongsil University, Seoul 156-743, Korea. "E-mail: kpaek@ssuac.k \\ Received August 18,2008
}

Key Words : Container molecule, Carceplex, Functionalization, Supranolecule

The selective functionalizations of container molecules are important tasks to expand its research fields. In case of covalent-bonded container molecules based on resorcin[4]arene their functionalization methods are quite limited compared to those for molecular capsules or calixarenes.

The functionalizations on tropical region of resorcin[4]arene-based container molecules with $D_{4 \mathrm{t}}$ symmetry have been usually accomplished by applying functionalized bridging reagent at the final convergent shell-closing step, which were successful for the manipulation of intrinsic properties of cavity. ${ }^{l}$ the solubility control. ${ }^{2}$ or the incorporation of metal ligands for multidimensional assembly of host. $^{3}$ The step-wise shell-closing reactions were also successful for $C_{4 v}$ or less symmetric carceplexes, ${ }^{+}$hemicarceplexes ${ }^{5}$ or cavitands ${ }^{6}$, which are mostly functionalized on their tropical regions.

The homogeneous four-feet functionalizations of $C_{4 v}$ resorcin[4]arene gave various functional container molecules such as monolayer of carceplex, ${ }^{7 b}$ metal-coordinated oligomers, ${ }^{7 \mathrm{c}}$ and water soluble molecular capsules. ${ }^{7 \mathrm{~d}}$ Especially the selective monofunctionalization methods on a foot ${ }^{8 a \mathrm{~b} b}$ of resorcin[4]arene gave the important intermediates for the new kinds of self-assemblies such as $\pi-\pi$ stacked oligomers, ${ }^{8 b_{1} 9 \mathrm{at}}$ star-shaped supramolecular polymer, ${ }^{9 \mathrm{~b}}$ and selfsorting hexameric capsules. ${ }^{*}$ Here are reported carceplexes 6 and 7 having a $p$-bromophenyl foot which can be used as a platform for the molecular engineering of carceplexes.

The key compound 1, p-bromophenyl tetramethyloctol, was obtained by the hetero condensation among 2-methylresorcinol, 4-bromobenzaldehyde, and octanal in about $40 \%$ yield. ${ }^{8 b}$ Tetramethylcavitand 2 was obtained in $33 \%$ yield by the 4-fold intramolecular cyclization of octol 1 using $\mathrm{CH}_{2} \mathrm{BrCl}$ and $\mathrm{K}_{2} \mathrm{CO}_{3}$. Tetramethylcavitand 2 was converted to $p$-bromophenyl tetrakis (bromonethyl)cavitand 3 by NBS/ AIBN in $42 \%$ yield.

The shell-closing reaction between $p$-bromophenyl tetrakis(bromomethyl)cavitand $\mathbf{3}$ and tetrakis(thiomethyl)cavitand $\mathbf{4}$ in a mixture of methyl ethyl ketone (MEK) or $N, N$-dimethylformamide (DMF) and $\mathrm{Rb}_{2} \mathrm{CO}_{3}$ gave $\mathrm{MEK} @$ carcerand 5 (carceplex 6) or DMF (a) carcerand 5 (carceplex 7) in $56 \%$ and $25 \%$ yield, respectively. The structures of $\operatorname{MEK}(\bar{a}$; carcerand $\mathbf{5}$ and DMF $(\bar{a}$; carcerand $\mathbf{5}$ were characterized with

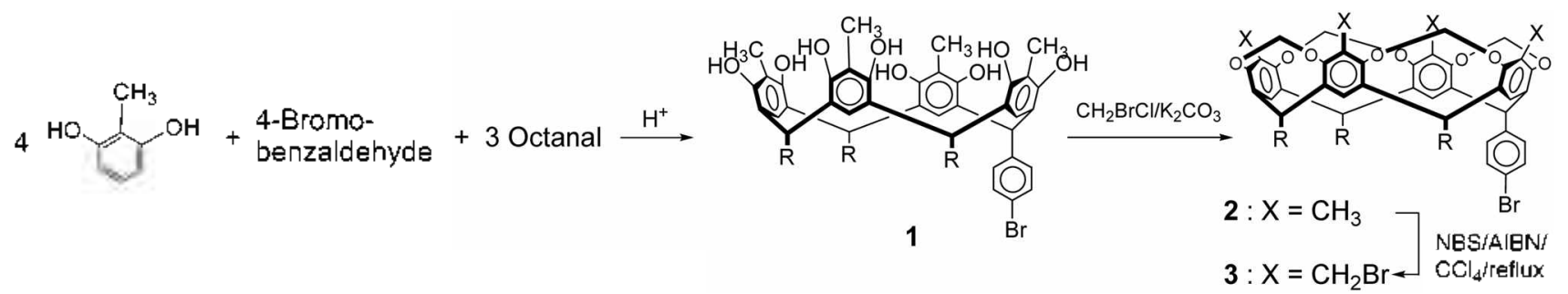

Scheme 1. Synthesis of $p$-Bromophenylcavitand $3(\mathrm{R}=\mathrm{Heptyl})$.

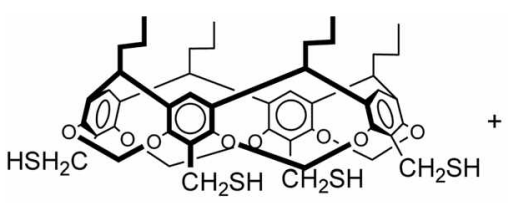

4

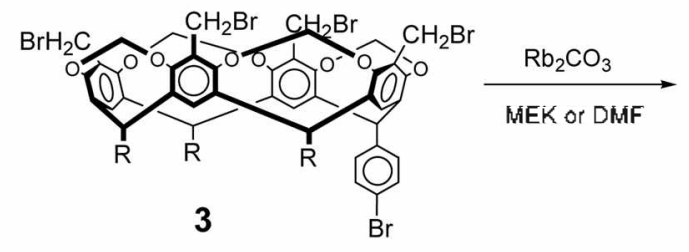

3

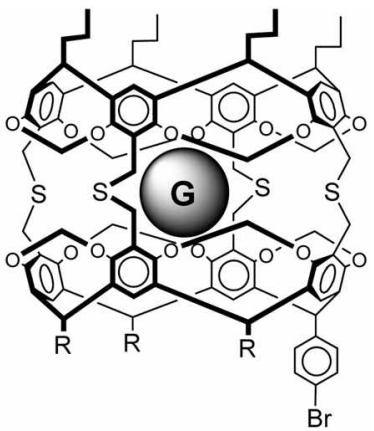

6:MEK@carcerand 5

7 : DMF@carcerand 5

Scheme 2. Synthesis of carceplexes 6 and $7(\mathrm{R}=$ Heptyl) having a functionalized foot. 
Table 1. 'H NMR Chemical shift changes of complexed guests

\begin{tabular}{ccccc}
\hline \multirow{2}{*}{ Guest structure } & & \multicolumn{3}{c}{ chemical shift $(\delta, \mathrm{ppm})$} \\
\cline { 2 - 5 } & & free & carceplex 6 or 7 & $\Delta \delta$ \\
\hline \multirow{3}{*}{$\mathrm{CH}_{3,0} \mathrm{COCH}_{2 .} \mathrm{CH}_{3 .}$} & $\mathrm{H}_{3}$ & 2.10 & -1.89 & 3.99 \\
& $\mathrm{H}_{\mathrm{b}}$ & 2.45 & 0.78 & 1.67 \\
& $\mathrm{H}_{\mathrm{s}}$ & 1.05 & -3.02 & 4.07 \\
\hline $\mathrm{H}_{\mathrm{L}}-\mathrm{C}(\mathrm{O}) \mathrm{N}-\mathrm{CH}_{3.2}$ & $\mathrm{H}_{\mathrm{b}}$ & 8.02 & 5.86 & 2.16 \\
$\mid$ & $\mathrm{H}_{\mathrm{c}}$ & 2.98 & 0.01 & 2.97 \\
$\mathrm{CH}_{3 \mathrm{r}}$ & $\mathrm{H}_{\mathrm{f}}$ & 2.89 & -0.23 & 3.12 \\
\hline
\end{tabular}

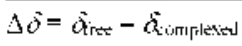

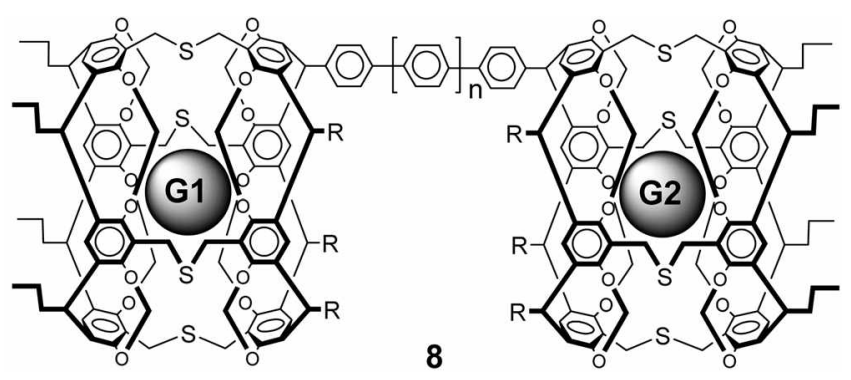

Figure 1. Biscarceplex 8 connected through a oligophenylene foot.

\section{MALDI TOF Mass and ${ }^{1} \mathrm{H} \times \mathrm{MR}$ spectrometry.}

Carceplexes 6 and 7 have a US football-shaped cavity whose dimensions are roughly $11 \AA \times 6 \AA$. The complexed linear guest tends to orient through the long axis of cavity in which guest's terminal hydrogen nests deep into the two poles of host where the magnetic shielding effects from the four benzene units are overlapped. Table 1 shows the chemical shift changes of hydrogens of incarcerated guests. MEK is more linear compared to DMF, so the terminal methyls of MEK nest proximal to the poles to give the two largest $\Delta \delta$ of 3.99 and 4.07 . The larger $\Delta \delta$ of $\mathrm{H}_{c}, 4.07$, than that of $\mathrm{H}_{\mathrm{a}}, 3.99$, is also due to their steric effect difference.

Such intrinsic properties of monomeric carceplexes have been extensively studied, ${ }^{1}$ but the studies on the cooperative multi-properties of dimer, oligomer, network, micelle, or membrane of container molecules are yet left to be explored. Figure 1 shows a prototypical biscarceplex 8 , two different carceplexes connected through a oligophenylene foot, which can be obtained from carceplexes 6,7 , or their analogues. The interactions between two incarcerated guests through oligophenylene bridge would change the electric or magnetic properties and the redox potentials, which could influence the relative orientational preferences or the stabilities of incarcerated guests. Such an interesting study will open a new era of supramolecular chemistry.

\section{Experimental Section}

Materials and General Procedures. All chemicals were reagent grades and directly used unless otherwise specified. All anhydrous reactions were conducted under an argon atmosphere. Melting points were determined using an Electrothermal LA9100 apparatus without calibration. MALDI-TOF
Mass spectra were run on a Voyager-DE ${ }^{\mathrm{TM}}$ STR Biospectrometry at National Center for Inter University Research Facilities (Seoul National University). The ${ }^{1} \mathrm{H}$ NMR spectra were recorded on a Brucker Avance $(400 \mathrm{MHz})$ spectrometer in $\mathrm{CDCl}_{3}$ unless stated otherwise. Gravity column chromatography was performed on silica gel 60 (E. Merck, 70-230 mash ASTM).

p-Bromophenyl Tetramethylcavitand 2. A mixture of octol $1(10 \mathrm{~g}, 10 \mathrm{mmol}), \mathrm{CH}_{2} \mathrm{BrCl}(3.4 \mathrm{~mL}, 50 \mathrm{mmol})$, $\mathrm{K}_{2} \mathrm{CO}_{3}(8.5 \mathrm{~g})$, and DMF $(50 \mathrm{~mL})$ was stirred at $50{ }^{\circ} \mathrm{C}$ overnight. The mixture was partitioned between $3 \times \mathrm{HCl}$ $(100 \mathrm{~mL})$ and $\mathrm{CH}_{2} \mathrm{Cl}_{2}(50 \mathrm{~mL})$, and the organic phase was separated, washed with $3 \times \mathrm{HCl}$, brine, and then dried over $\mathrm{MgSO}_{4}$. The solvent was evaporated and the residue was purified by silica gel chromatography using a mixture of EtOAc/Hexane (1:11). The best fractions were collected, concentrated, and the residue was recrystallized in a mixture of $\mathrm{CH}_{3} \mathrm{CN} / \mathrm{CH}_{2} \mathrm{Cl}_{2}$ to give a product $(3.5 \mathrm{~g}, 33 \%$ ); mp 145 $148{ }^{\circ} \mathrm{C}$; MALDI TOF MS m/z $1041.58\left(100 \%, \mathrm{M}^{+}\right) ;{ }^{1} \mathrm{H}$ NMR (400 MHz, CDCl $\left.1_{3}\right) \delta 0.86\left(9 \mathrm{H}, \mathrm{m}, \mathrm{CH}_{2}\left(\mathrm{CH}_{2}\right)_{5} \mathrm{CH}_{3}\right)$, $\left.1.25-1.52\left(30 \mathrm{H}, \mathrm{m}, \mathrm{CH}_{2}\left(\mathrm{CH}_{2}\right)_{5} \mathrm{CH}_{3}\right), 1.97(6 \mathrm{H}, \mathrm{s}, \mathrm{ArCH})_{3}\right)$, $2.02(6 \mathrm{H}, \mathrm{s}, \mathrm{ArCH}), 2.11\left(6 \mathrm{H}, \mathrm{bs}, \mathrm{CH}_{2}\left(\mathrm{CH}_{2}\right)_{5} \mathrm{CH}_{3}\right), 4.22$ $\left(1 \mathrm{H}, \mathrm{d}, J=7.2 \mathrm{~Hz}\right.$, inner $\left.\mathrm{OCH}_{2} \mathrm{O}\right), 4.28(2 \mathrm{H}, \mathrm{d}, J=7.2 \mathrm{~Hz}$, inner $\left.\mathrm{OCH}_{2} \mathrm{O}\right), 4.33\left(\mathrm{HH}, \mathrm{d}, J=6.8 \mathrm{~Hz}\right.$, inner $\left.\mathrm{OCH}_{2} \mathrm{O}\right), 4.75$ $(3 \mathrm{H}, \mathrm{m}, \mathrm{CH}$ methine), $5.91(4 \mathrm{H}, \mathrm{m}$, outer $\mathrm{OCH}, \mathrm{O}), 6.34$ $(1 \mathrm{H}, \mathrm{s}, \mathrm{CH}$ methine), 6.86 and 6.95 (two $2 \mathrm{H}$, two s, $\mathrm{Ar} H$ ), $7.29(2 \mathrm{H}, \mathrm{d}, J=8.2 \mathrm{~Hz}, p$-bromophenyl), $7.49(2 \mathrm{H}, \mathrm{d}, J=8.2$ $\mathrm{Hz}, p$-bromophenyl); ${ }^{13} \mathrm{C} \mathrm{XMR}\left(100 \mathrm{MHz}, \mathrm{CDCl}_{3}\right) \delta 9.4$, $9.5,13.2,13.3,21.8,21.9,27.0,27.3,28.5,28.7,28.8,29.0$, $29.3,30.9,31.1,36.1,36.2,41.3,97.7,116.8,119.3,119.7$, $122.8,123.3,130.2,135.8,137.0,137.2,139.0,152.3,153.1$.

p-Bromophenyl Tetrakis(bromomethyl)cavitand 3. A mixture of cavitand 2 ( $2 \mathrm{~g}, 2 \mathrm{mmol})$, NBS ( $\mathrm{g}, 11 \mathrm{mmol}$ ), AIBN ( $2 \mathrm{mg})$, and $\mathrm{CCl}_{4}(50 \mathrm{~mL})$ was refluxed for $5 \mathrm{hrs}$. The mixture was cooled to rt and the solvent was evaporated The residue was partitioned between water $(100 \mathrm{~mL})$ and $\mathrm{CH}_{2} \mathrm{Cl}_{2}(50 \mathrm{~mL})$, and the organic phase was separated, washed with brine, and then dried over $\mathrm{MgSO}_{4}$. The solvent was evaporated and the residue was purified by silica gel chromatography using a 2:1 mixture of $\mathrm{Hexane} / \mathrm{CH}_{2} \mathrm{Cl}_{2}$. The best fractions were collected, concentrated, and the residue was recrystallized in a mixture of $\mathrm{CH}_{2} \mathrm{Cl}_{2} / \mathrm{EtOH}$ to give a product $(1.08 \mathrm{~g}, 42 \%) ; \mathrm{mp} 192-195{ }^{\circ} \mathrm{C}$; MALDI TOF MS $\mathrm{m} / \mathrm{z} 1277.31\left(100 \%,[\mathrm{M}-\mathrm{Br}]^{-}\right), 1357.23\left(50 \%, \mathrm{M}^{+}\right)$; ${ }^{1} \mathrm{H} \mathrm{NMR}$ $\left(400 \mathrm{MHz}, \mathrm{CDCl}_{3}\right) \delta 0.90\left(9 \mathrm{H}, \mathrm{m}, \mathrm{CH}_{2}\left(\mathrm{CH}_{2}\right)_{5} \mathrm{CH}_{3}\right), 1.24-1.32$ $\left(30 \mathrm{H}, \mathrm{m}, \mathrm{CH}_{2}\left(\mathrm{CH}_{2}\right)_{5} \mathrm{CH}_{3}\right), 2.09\left(6 \mathrm{H}, \mathrm{bs}, \mathrm{CH}_{2}\left(\mathrm{CH}_{2}\right)_{5} \mathrm{CH}_{3}\right)$, $4.42\left(4 \mathrm{H}, \mathrm{s}, \mathrm{ArCH} \mathrm{Br}_{2} \mathrm{Br}\right), 4.47\left(6 \mathrm{H}, \mathrm{s}, \mathrm{ArCH}_{2} \mathrm{Br}\right), 4.60(4 \mathrm{H}, \mathrm{m}$, inner $\left.\mathrm{OCH}_{2} \mathrm{O}\right), 4.75(3 \mathrm{H}, \mathrm{m}, \mathrm{CH}$ methine $), 6.04(4 \mathrm{H}, \mathrm{m}$, outer $\mathrm{OCH}_{2} \mathrm{O}$ ), $6.34(\mathrm{lH}, \mathrm{s}, \mathrm{CH}$ methine), 7.00 and 7.10 (two $2 \mathrm{H}$, two s, $\operatorname{Ar} H), 7.21(2 \mathrm{H}, \mathrm{d}, J=8.4 \mathrm{~Hz}, p$-bromophenyl), $7.51\left(2 \mathrm{H}, \mathrm{d}, J=8.4 \mathrm{~Hz}, p\right.$-bromophenyl); ${ }^{13} \mathrm{C}$ NMR $(100$ $\mathrm{MHz}, \mathrm{CDCl}_{3}$ ) $\delta 14.1,22.6,22.7,22.9,27.8,28.1,29.3,29.5$, $29.8,30.2,31.8,31.9,36.9,41.9,99.2,121.0,123.9,124.5$, $125.0,130.9,131.4,136.8,137.9,138.3,138.8,153.4,154.3$.

Tetrakis(thiomethyl)eavitand 4. A mixture of tetrakis(bromomethyl)cavitand ${ }^{10}$ ( $1.0 \mathrm{~g}, 0.93 \mathrm{mmol}$ ), thiourea (566 mg, $7.4 \mathrm{mmol}$ ), and $\mathrm{DMF}$ ( $(5 \mathrm{~mL}$ ) was stirred for 3 hrs at 60 
${ }^{\circ} \mathrm{C}$ and then for $2 \mathrm{hrs}$ at $80^{\circ} \mathrm{C}$ under argon. To the reaction mixture $4 \mathrm{~mL}$ of $3 \mathrm{~N} \mathrm{NaOH}$ was added and stirred for $30 \mathrm{~min}$ and then $5 \mathrm{~mL}$ of $3 \times \mathrm{HCl}$ was added. The product was extracted with $20 \mathrm{~mL}$ of $\mathrm{CH}_{2} \mathrm{Cl}_{2}$ and the organic phase was washed with water and brine, and then dried over $\mathrm{MgSO}_{4}$. The solvent was evaporated and the residue was recrystallized in a mixture of hexane and $\mathrm{CH}_{2} \mathrm{Cl}_{2}$ to give a product (light pink powder, $542 \mathrm{mg}, 66 \%$ ); $\mathrm{mp}>138^{\circ} \mathrm{C}$ (decomposed); ${ }^{1} \mathrm{H} \backslash \mathrm{MR}(400 \mathrm{MHz}, \mathrm{CDCl}) \delta 1.02(12 \mathrm{H}, \mathrm{t}, J=8.0 \mathrm{~Hz}$, $\left.\mathrm{CH}_{2} \mathrm{CH}_{2} \mathrm{CH}_{3}\right), 1.39\left(8 \mathrm{H}, \mathrm{m}, \mathrm{CH}_{2} \mathrm{CH}_{2} \mathrm{CH}_{3}\right), 1.87(4 \mathrm{H}, \mathrm{t}, J=$ $8.0 \mathrm{~Hz}, \mathrm{SH}), 2.21\left(8 \mathrm{H}, \mathrm{m}, \mathrm{CH}_{2} \mathrm{CH}_{2} \mathrm{CH}_{3}\right), 3.58(8 \mathrm{H}, \mathrm{d}, J=8.0$ $\left.\mathrm{Hz}, \mathrm{CH}_{2} \mathrm{SH}\right), 4.46\left(1 \mathrm{H}, \mathrm{d}, J=8.0 \mathrm{~Hz}\right.$, inner $\left.\mathrm{OCH} \mathrm{H}_{2} \mathrm{O}\right), 4.78$ ( $4 \mathrm{H}, \mathrm{t}, J=8.0 \mathrm{~Hz}, C H$ methine), $5.96(4 \mathrm{H}, \mathrm{d}, J=8.0 \mathrm{~Hz}$, outer $\left.\mathrm{OCH}_{2} \mathrm{O}\right), 7.07(4 \mathrm{H}, \mathrm{s}, \mathrm{Ar} H) ;{ }^{13} \mathrm{C} \mathrm{NMR}\left(100 \mathrm{MHz}, \mathrm{CDCl}_{3}\right) \delta$ $14.5,18.5,21.3,32.5,37.0,100.3,119.7,127.6,138.4,153.5$.

MEK $(a p$-Bromophenyl Carcerand 5 (Carceplex 6). A solution of $p$-bromophenyl tetrakis(bromomethyl)cavitand 3 (300 $\mathrm{mg}, 0.22 \mathrm{mmol}$ ), tetrakis(thiomethyl)cavitand 4 (196 mg. $0.22 \mathrm{mmol}$ ), and MEK (100 mL) was added for $12 \mathrm{hrs}$ to a refluxing mixture of $\mathrm{Rb}_{2} \mathrm{CO}_{3}(1.02 \mathrm{~g})$ and $\mathrm{MEK}(100$ $\mathrm{mL})$ in a $500 \mathrm{~mL}$ Round-bottom flask. After additional 12 hrs refluxing, the solvent was evaporated and the residue was partitioned between water and $\mathrm{CH}_{2} \mathrm{Cl}_{2}$. The organic phase was separated and washed with brine, and then dried over $\mathrm{MgSO}_{4}$. The solvent was evaporated and the residue was purified by silica gel chromatography using a 1:3 mixture of hexane/ $/ \mathrm{CH}_{2} \mathrm{Cl}_{2}$. The best fractions were collected, concentrated, and the residue was recrystallized in a mixture of $\mathrm{CH}_{3} \mathrm{Cl} / \mathrm{CH}_{3} \mathrm{CX}$ to give a product (White powder, $245 \mathrm{mg}$, $56 \%$ ); mp $>190^{\circ} \mathrm{C}$ (decomposed); MALDI TOF MS m/z $1992.82\left(15 \%, \mathrm{M}^{-}\right)$; ${ }^{\mathrm{l}} \mathrm{H}$ NMR $\left(400 \mathrm{MHz}, \mathrm{CDCl}_{3}\right) \delta-3.02$ $\left(3 \mathrm{H}, \mathrm{m}, \mathrm{CH}_{3} \mathrm{CH}_{2}\right.$ of $\left.\mathrm{MEK}\right),-1.89\left(3 \mathrm{H}, \mathrm{m}, \mathrm{CH}_{3} \mathrm{CO}\right.$ of MEK). $0.78\left(2 \mathrm{H}, \mathrm{m}, \mathrm{CH}_{3} \mathrm{CH}_{2}\right.$ of $\left.\mathrm{MEK}\right), 0.88(9 \mathrm{H}, \mathrm{m}, 3 \mathrm{x}$ $\left.\mathrm{CH}_{2}\left(\mathrm{CH}_{2}\right)_{5} \mathrm{CH}_{3}\right), 1.02\left(12 \mathrm{H}, \mathrm{t}, J=8 \mathrm{~Hz}, 4 \times \mathrm{CH}_{2} \mathrm{CH}_{2} \mathrm{CH}_{3}\right)$, $1.25-1.59\left(38 \mathrm{H}, \mathrm{m}, 3 \times \mathrm{CH}_{2}\left(\mathrm{CH}_{2}\right)_{5} \mathrm{CH}_{3}+4 \times \mathrm{CH}_{2} \mathrm{CH}_{2} \mathrm{CH}_{3}\right)$, $2.00-2.20\left(14 \mathrm{H}, \mathrm{m}, 3 \times \mathrm{CH}_{2}\left(\mathrm{CH}_{2}\right)_{5} \mathrm{CH}_{3}+4 \times \mathrm{CH}_{2} \mathrm{CH}_{2} \mathrm{CH}_{3}\right)$, $3.88-3.92\left(16 \mathrm{H}, \mathrm{m}, 4 \times \mathrm{CH}_{2} \mathrm{SCH} \mathrm{H}_{2}\right), 4.73(3 \mathrm{H}, \mathrm{m}, \mathrm{CH}$ methine $)$, 4.66-4.75 $\left(12 \mathrm{H}, \mathrm{m}, 8 \times\right.$ inner $\mathrm{OCH}_{2} \mathrm{O}+4 \times \mathrm{CH}$ methine), $5.90\left(8 \mathrm{H}, \mathrm{m}\right.$, outer $\left.\mathrm{OCH}_{2} \mathrm{O}\right), 6.29(1 \mathrm{H}, \mathrm{s}, \mathrm{CH}$ methine), 6.91 and 7.00 (two $2 \mathrm{H}$, two s, $\mathrm{Ar} H$ ), $7.06(4 \mathrm{H}, \mathrm{s}$, ArH $), 7.20(2 \mathrm{H}, \mathrm{d}, J=8.0 \mathrm{~Hz}, p$-bromophenyl), $7.48(2 \mathrm{H}, \mathrm{d}$, $J=8.0 \mathrm{~Hz}, p$-bromophenyl).

DMF(ap-Bromophenyl Carcerand 5 (Carceplex 7). A solution of $p$-bromophenyl tetrakis(bromomethyl)cavitand 3 (300 $\mathrm{mg}, 0.22 \mathrm{mmol}$ ), tetrakis(thiomethyl)cavitand 4 (196 $\mathrm{mg}, 0.22 \mathrm{mmol})$, and DMF $(100 \mathrm{~mL})$ was added for $12 \mathrm{hrs}$ to a mixture of $\mathrm{Rb}_{2} \mathrm{CO}_{3}(1.02 \mathrm{~g})$ and $\mathrm{DMF}(100 \mathrm{~mL})$ at $80^{\circ} \mathrm{C}$ in a $500 \mathrm{~mL}$ Round-bottom flask. After additional $12 \mathrm{hrs}$ stirring at $80^{\circ} \mathrm{C}$, the reaction mixture was cooled to room temperature and then partitioned between $3 \mathrm{~N} \mathrm{HCl}(150 \mathrm{~mL})$ and $\mathrm{CH}_{2} \mathrm{CH}_{2}(150 \mathrm{~mL})$. The aqueous phase was extracted with $50 \mathrm{~mL}$ of $\mathrm{CH}_{2} \mathrm{CH}_{2}$ and the combined organic phase was washed with $100 \mathrm{~mL}$ of $3 \mathrm{~N} \mathrm{HCl}$. The organic phase was separated and washed with brine, and then dried over
$\mathrm{MgSO}_{4}$. The solvent was evaporated and the residue was purified by silica gel chromatography using a 1:3 mixture of hexane $/ \mathrm{CH}_{2} \mathrm{Cl}_{2}$. The best fractions were collected, concentrated, and the residue was recrystallized in a mixture of $\mathrm{CH}_{3} \mathrm{Cl} / \mathrm{CH}_{3} \mathrm{CN}$ to give a product (White powder, $112 \mathrm{mg}$, $25 \%$ ); mp $>190^{\circ} \mathrm{C}$ (decomposed); MALDI TOF MS m/z 1923.89 ([M-DMF] $\left.{ }^{+}, 90 \%\right), 1940.88$ ([M-DMF $\left.+\mathrm{H}_{2} \mathrm{O}\right]^{-}$, $100 \%), 1996.91\left([\mathrm{M}]^{+}, 25 \%\right) ;{ }^{1} \mathrm{H} \times \mathrm{MR}\left(400 \mathrm{MHz}, \mathrm{CDCl}_{3}\right) \delta$ $-0.23(3 \mathrm{H}, \mathrm{s}, \mathrm{N}-\mathrm{CH}), 0.01\left(3 \mathrm{H}, \mathrm{s}, \mathrm{N}-\mathrm{CH} \mathrm{H}_{3}\right), 0.90-1.07(9 \mathrm{H}$, m, $\left.3 \times \mathrm{CH}_{2}\left(\mathrm{CH}_{2}\right)_{5} \mathrm{CH}_{3}\right), 0.95(12 \mathrm{H}, \mathrm{t}, J=8 \mathrm{~Hz}, 4 \mathrm{x}$ $\left.\mathrm{CH}_{2} \mathrm{CH}_{2} \mathrm{CH}_{3}\right), 1.32-1.45\left(38 \mathrm{H}, \mathrm{m}, 3 \times \mathrm{CH}_{2}\left(\mathrm{CH}_{2}\right)_{5} \mathrm{CH}_{3}+4 \mathrm{x}\right.$ $\left.\mathrm{CH}_{2} \mathrm{CH}_{2} \mathrm{CH}_{3}\right), 2.06-2.24\left(14 \mathrm{H}, \mathrm{m}, 3 \times \mathrm{CH}_{2}\left(\mathrm{CH}_{2}\right)_{5} \mathrm{CH}_{3}+4 \mathrm{x}\right.$ $\left.\mathrm{CH}_{2} \mathrm{CH}_{2} \mathrm{CH}_{3}\right), 3.93-3.98\left(16 \mathrm{H}, \mathrm{m}, 4 \times \mathrm{CH}_{2} \mathrm{SCH}\right)_{2}, 4.41-4.83$ $\left(15 \mathrm{H}, \mathrm{m}, 3 \mathrm{x} C H\right.$ methine $+8 \times$ inner $\mathrm{OCH}_{2} \mathrm{O}+4 \times \mathrm{CH}$ methine), 5.86-5.95 (8H, m, outer OCH.O $), 6.34(1 \mathrm{H}, \mathrm{s}, \mathrm{CH}$ methine), 7.01 and 7.08 (two $2 \mathrm{H}$, two s, $\mathrm{ArH}), 7.09(4 \mathrm{H}, \mathrm{s}$, ArH), $7.27(2 \mathrm{H}, \mathrm{d}, J=8.0 \mathrm{~Hz}, p$-bromophenyl), $7.54(2 \mathrm{H}, \mathrm{d}$, $J=8.0 \mathrm{~Hz}, p$-bromophenyl).

Acknowledgments. The financial support from $\mathrm{CBMH}$ (Yonsei Lniversity), Seoul R\&BD Program, and Soongsil University is acknowledged.

\section{References}

1. Cram, D. J.; Cram, J. M. Contriner Molecules cmol Their Guests, Monographs in Stiprantolecular Chemistry; Stoddart, J. F., Ed.; The Royal Society of Chemistry Press: Cambridge, 1994; Vol. 4.

2. (a) Yoon, J.; Cram, D.J. Chem. Commen. 1997, 497. (b) Park, B. S.; Knobler, C. B.; Eid, C. N., Jr.; Warmuth, R.; Cram, D. J. Chem. Commm 1998, 55

3. Chang, T.-Y.; Paek, K. Bull. Kor: Chem. Soc. 2008, 29, 276.

4. (a) Timmerman, P.; Verboom, W; van Veggel, F. C. J. M.; van Duynhoven, J. P. M.; Reinhoudt, D. N. Angen: Chem. Int. Ed. Engl. 1994, 33, 2345. (b) llum, C.; Jo, E.; Kim, J.; Paek, K. Angew: Chem. Int. Ed. 2006, 45, 2056.

5. (a) Yoon, J.; Knobler, C. B.; Maverick, E. F.; Cram, D. J. Chem. Conmmin 1997, 1303. (b) Paek, K.; Ihm, H.; Yun, S.; Lee, H. C.; No, K. T. J. Org. Chen. 2001, 66, 5736.

6. (a) Yoon, Y.-S.; Park, H. S.; Paek, K. Bull. Kor: Chen. Soc. 2006, 27, 2055. (b) Ye, B.; Paek, K. Bull. Kor: Chem. Soc. 2006, 27, 305. (c) Ilm, H.; Hwang, S.-J.; Prek, K. Tetrahedron Letr. 2004, 45, 9119

7. (a) Gibb, B. C.; Chapman, R. G.; Shenman, J. C. J. Org. Chem. 1996, 61, 1505. (b) Huisman, B.-H.; Rudkevich, D. M.; van Veggel, F. C. J. M.; Reinhoudt, D. N. J. Am. Chem. Soc. 1996, 118, 3523. (c) Pirondini, L.; Stendardo, A. G.; Geremia, S.; Campagnolo, M.; Samori, P.; Rabe, J. P.; Fokkens, R.; Dalcanale, E. Angew: Chem. Int. Ed. 2003, 42, 1384. (d) Natarajau, A. Kaanumalle, L. S.; Jockusch, S.; Gibb, C. L. D.; Gibb, B. C. Turro, N. J.; Ramamurthy, V. J. An. Chem. Soc. 2007, 129, 4132.

8. (a) Saito, S.; Rudkevich, D. M.; Rebek, J. Jr. Org. Lett. 1999, 1 , 1241. (b) Ihm, H.; Ahu, J.-S.; Lah, M. S.; Koh, Y. H.; Paek, K. Org. Lett. 2004, 6, 3893. (c) Hauke, F.; Myles, A. J.; Rebek, J., Jr. Chem. Commu. 2005, 4164. (d) Barrett, E. S.; Dale, T. J.; Rebek. J., Jr. J. Am. Chem. Soc. 2007, 129, 3818.

9. (a) Kwak, M.-J.; Paek, K. Bull. Kor: Chem. Soc. 2007, 28, 1440. (b) Yebeutchou, R. M.; Tancini, F;; Demitri, N.; Geremia, S.; Mendichi, R.; Dalcanale, E. Angen: Chem. Int. Ed. 2008, 47, 4504. (c) Barrett, E. S.; Dale, T. J.; Rebek, J., Jr. J. Am. Chem. Soc. 2008, $130,2344$.

10. Kim, K.; Prek, K. Bull. Korean Chem. Soc. 1993, 14, 658. 